\title{
Endobronchial ultrasound and positron emission tomography positive mediastinal
} lymph nodes

\author{
G. Plat*, P. Pierard*, A. Haller" ${ }^{\#}$ J. Hutsebaut*, J. Faber*, M. Dusart ${ }^{\ddagger}$, P. Eisendrath*, \\ J-P. Sculier* and V. Ninane ${ }^{+}$
}

ABSTRACT: Positron emission tomography with 18F-fluoro-2-deoxy-D-glucose (FDG-PET) is more accurate than computed tomography for staging of mediastinal (hilar) lymph nodes. In the case of positive findings, tissue sampling of lymph nodes is required. The diagnostic/staging yield of transbronchial needle aspiration (TBNA) following endobronchial ultrasound (EBUS) localisation was assessed in this particular clinical setting. The number of avoided surgical procedures was evaluated.

All consecutive patients referred for staging and/or diagnosis of mediastinal FDG-PET positive lesions were included. Data were prospectively collected. TBNA sampling of lymph nodes was performed after EBUS localisation. If no diagnosis was reached, further surgical sampling or adequate follow-up was performed.

From January 2003 to June 2004, 33 patients were included. The average number of TBNA samples per patient was $4.2 \pm 1.5$. Cytological or histological diagnoses were obtained in 27 (82\%) of the patients, of which $78 \%$ were obtained after previous EBUS localisation. In 25 (76\%) of the 33 patients, surgical staging procedures were suppressed.

In conclusion, transbronchial needle aspiration after endobronchial ultrasound localisation should be considered as a primary method of evaluation of lymph nodes positive by positron emission tomography with 18F-fluoro-2-deoxy-D-glucose, and may replace the majority of surgical mediastinal staging/diagnostic procedures.

KEYWORDS: Endobronchial ultrasound, lung cancer, mediastinal lymphadenopathy, positron emission tomography scan, staging, transbronchial needle aspiration

ung cancer remains the first cause of cancer-related deaths, with an overall 5yr survival rate of $15 \%$. The best hope for cure is for patients with potentially resectable disease, but even in these cases, the 5-yr survival rate is only in the order of $40-50 \%$ [1]. Adequate staging before surgery is, thus, of paramount importance to better stratify the therapeutic approach and to limit the number of futile thoracotomies. In particular, patients with N2 mediastinal lymph node involvement are poor candidates for initial surgical resection, since 5-yr survival rate in this condition ranges, according to various series, from $11-30 \%$ only [2].

Computed tomography (CT) has traditionally been used to predict lymph node involvement, and a short axis of lymph node exceeding $1 \mathrm{~cm}$ has been chosen to indicate a high probability of neoplastic involvement. Using this criterion, however, the sensitivity and specificity of CT are only 0.57 and 0.82 , respectively [3]. Recently, the positron emission tomography with $18 \mathrm{~F}$ fluoro-2-deoxy-D-glucose (FDG-PET) has been shown to be more accurate than $\mathrm{CT}$ in the evaluation of mediastinal lymph node involvement [4]. It is now used in many countries, in addition to $\mathrm{CT}$, to further improve the sensitivity and specificity rates of the noninvasive assessment of mediastinal lymph nodes. This is reflected in recent consensus recommending that, in patients with enlarged mediastinal lymph nodes on CT scans who are potential candidates for surgery, a whole-body FDG-PET scan should be performed [5]. FDG-PET has a sensitivity of 0.85 , a specificity of 0.88 , a positive predictive value of 0.78 , and a negative predictive value of 0.93 [3]. It has then been suggested that a negative mediastinal FDG-PET result may obviate the need for invasive lymph node sampling [4], but a positive one needs to be confirmed [4,5].

\section{AFFLLIATIONS}

*Depts of Internal Medicine,

"Nuclear Medicine, Institut Bordet, and

\#Pathology, and

${ }^{+}$Chest Service, Saint-Pierre Hospital, Brussels, Belgium.

CORRESPONDENCE

V. Ninane

Chest Service

Saint-Pierre Hospital

Rue Haute 322

1000 Brussels

Belgium

Fax: 3225354174

E-mail: vincent_ninane@stpierre-

bru.be

Received:

December 072004

Accepted after revision:

October 132005 
In case of positive mediastinal FDG-PET results, further assessment using mediastinoscopy is still considered as the "gold-standard" [3]. Mediastinoscopy, however, is a surgical procedure with a mortality rate of $0.2 \%$ and a morbidity rate of $0.5-2.5 \%$ [6]. In addition, it requires general anaesthesia and at least one overnight stay in the hospital [6]. Transbronchial needle aspiration (TBNA) of lymph nodes, which was developed $>50$ yrs ago [7], may circumvent some of the drawbacks of mediastinoscopy. Surprisingly, this technique remains largely underutilised [8] and this is probably explained, at least in part, by concerns about the practice learning curve and by the fact that, until recently, this procedure was not guided by imaging. In a recent study, HERTH et al. [9] have shown that TBNA might be assisted by previous localisation of mediastinal lymph nodes using a new tool, endobronchial ultrasound (EBUS). They also showed that this strategy was associated with an increased diagnostic yield of TBNA.

The current authors' reasoned that the diagnostic yield of TBNA might be even higher if, in addition to lymph node sampling after EBUS localisation, evaluation was specifically performed in the lymph node areas showing abnormal results during FDG-PET scan. With this in mind, all patients referred to the endoscopic unit of Institut Bordet, Saint-Pierre Hospital (Brussels, Belgium), for diagnosis and/or staging of mediastinal lymph nodes with abnormal results of FDG-PET scan were assessed with EBUS followed by TBNA, and needle aspiration was concentrated in areas shown to be abnormal by FDG-PET. As a secondary aim, the current authors also tried to assess in which percentage of the cases this strategy might obviate the need for mediastinoscopy or other major surgical diagnostic/staging procedures.

\section{MATERIALS AND METHODS}

All the patients with FDG-PET positive lymph nodes referred to the endoscopic unit of Institut Bordet, Saint-Pierre Hospital, for evaluation were considered. Of them, 17 (52\%) were referred from eight other hospitals. All patients underwent EBUS examination for localisation of the FDG-PET abnormal lymph node area followed by TBNA. Data were prospectively collected and analysed to assess the diagnostic yield of TBNA in this particular setting. These patients had three different initial clinical presentations. In group I (staging), lung cancer was histologically confirmed, without any evidence for distant metastasis, and FDG-PET was suggestive of mediastinal lymph node involvement. Group II (diagnosis and staging) included patients with lesions that were suspected to correspond to lung cancer, but the initial bronchoscopy was not contributive and FDG-PET was abnormal at the level of the tumour and of the mediastinum. Group III (lymphadenopathy work-up) was more heterogeneous, and included patients with one or more FDG-PET positive lymph nodes without evidence of lung tumour.

Bronchoscopy was performed using a large working channel bronchoscope (Olympus Excera; Olympus, Tokyo, Japan) while patients were comfortably seated in a $30^{\circ}$ recumbent position in a high-backed armchair. Oxygen $\left(2 \mathrm{~L} \cdot \mathrm{min}^{-1}\right)$ was administered with nasal prongs, and transcutaneous haemoglobin saturation and cardiac rhythm (Ohmeda Biox 3740; Ohmeda, Louisville, CO, USA) were monitored continuously during the whole procedure. Analgesia of the airways was performed as previously described [10], with conscious sedation using intravenous midazolam; patients were evaluated on a real outpatient basis. A 20-MHz radial mechanical transducer type ultrasonic probe (UM-BS20-26R; Olympus) in a flexible sheet equipped with a balloon at the tip (Maj-643R; Olympus), connected to a EBUS processor (EU-M20; Olympus) was inserted in the channel and used to localise the abnormal FDG-PET lymph nodes and to make precise their relationship with the tracheobronchial tree. Examination was performed with the balloon inflated to optimise contact between the miniaturised probe and the bronchial wall. It was also performed with the small probe applied directly on the wall, without balloon inflation, attempting to better demarcate sampling location (fig. 1). The catheter was then pulled out
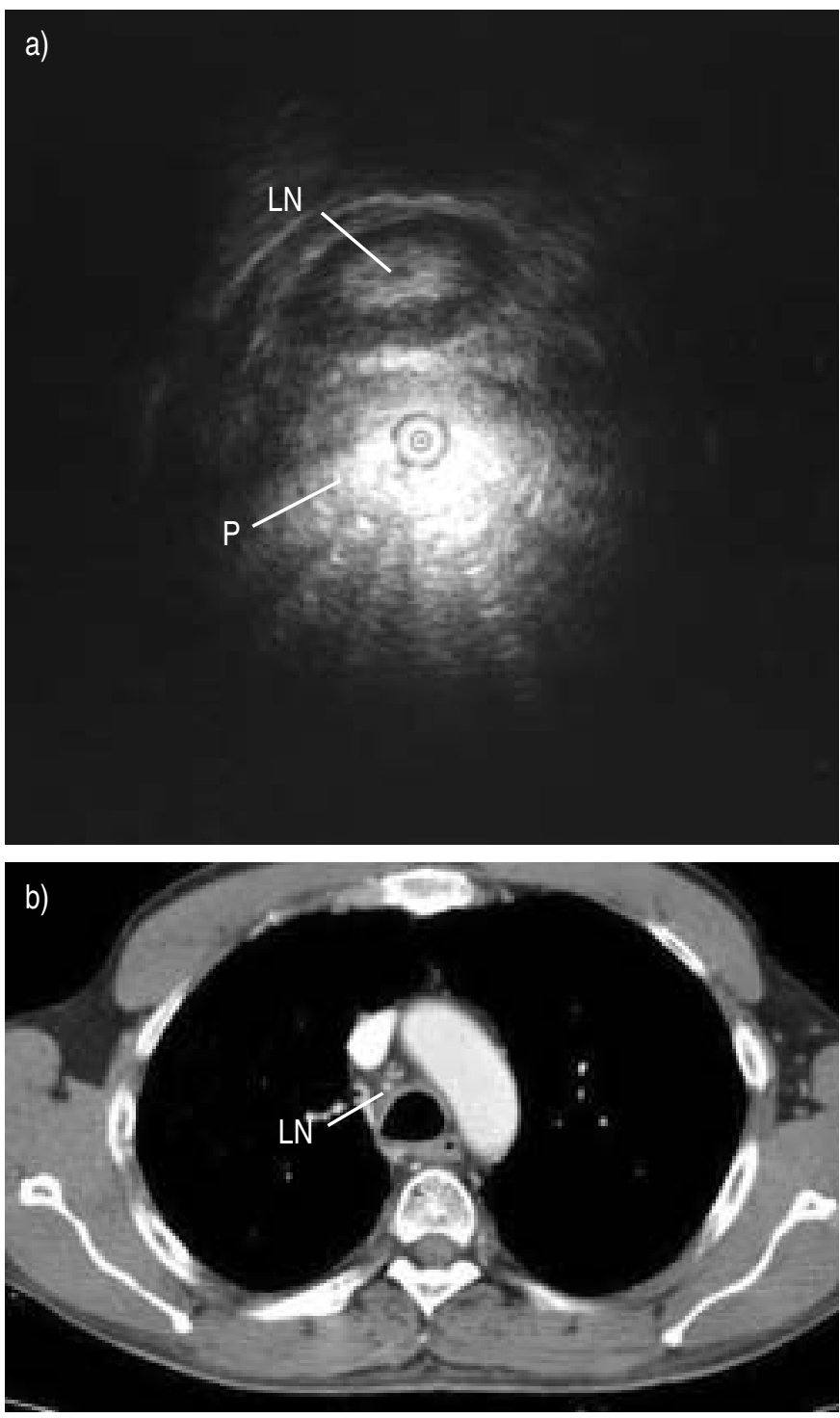

FIGURE 1. a) Endobronchial ultrasound image of a lymph node (LN) with a hypoechoic centre at level 4R. The balloon is not inflated and the small ultrasonic probe $(P$, diameter $2.5 \mathrm{~mm}$ ) is in direct contact with the tracheal wall. The small size of the probe, without balloon inflation, allows better demarcation of the sampling location. b) Computed tomography scan at level $4 \mathrm{R}$ showing two $\mathrm{LN}$, the largest corresponding to the lymph node represented in a). 
and a combined cytological and histological needle (BARDWang, Billerica, MA, USA) was systematically used for TBNA. In each patient, four to six punctures were performed, beginning with the highest staging node level [11,12] in the case where several areas were FDG-PET abnormal. In case of failure of EBUS to localise lymph nodes, sampling location was based on CT and PET scan information using the methodology previously described [11]. No rapid on-site cytological examination was used.

For the purpose of analysis, TBNA was considered as contributive whenever a clear definite cytological or histological diagnosis (including in the latter case normal lymphoid tissue) was obtained. It was not contributive if no diagnosis (including only rare suspicious cells without lymphocytes on cytological examination) could be reached. In these latter cases, surgical staging/diagnostic procedure was recommended and, if it was not performed, final diagnosis was then based on adequate clinical follow-up. A surgical staging/diagnostic procedure was considered as eluded whenever TBNA provided a positive result in groups I and II, and a definite diagnosis in group III, and on the condition that there was no alternative, less invasive method than surgery.

Unless otherwise specified, results are expressed as mean \pm SD. No comparison was made between the three different groups of patients, or between patients with successful or unsuccessful previous localisation with EBUS, because the numbers in the study were too low.

\section{RESULTS}

From January 2003 to June 2004, 33 patients with positive FDGPET mediastinal (hilar) lymph nodes were referred for TBNA exploration after previous evaluation with EBUS. Their main characteristics are summarised in table 1. The bronchoscopist could always look at the FDG-PET images and the CT scans, but data on quantitative assessment, including standardised uptake value (FDG-PET) or lymph node size (CT scan), were often missing due to nonstandardised initial diagnostic workup (nine different centres). The procedure, performed under local anaesthetic, was well tolerated and no significant sideeffect was encountered (with the exception of some agitation,

\begin{tabular}{lc}
\hline TABLE 1 & \\
\hline Characteristic & Patients \\
\hline & \\
Total number & 33 \\
$\quad$ Proven lung cancer & 5 \\
Suspected lung cancer & 19 \\
Isolated lymphadenopathy(ies) & 9 \\
Active or former smokers/nonsmokers & $29 / 4$ \\
Male/female & $19 / 14$ \\
Mean age yrs & $64.7 \pm 11.4$ \\
Hilar FDG-PET positive lymph nodes only & 3 \\
Mediastinal FDG-PET positive lymph nodes & 30 \\
\hline
\end{tabular}

Data are presented as mean \pm SD or $n$. FDG-PET: positron emission tomography with $18 \mathrm{~F}$-fluoro-2-deoxy-D-glucose. in one patient, that limited the number of needle punctures to one, and cough that occurred in some patients but without significantly disturbing examination and sampling). FDG-PET scan located abnormal lymph nodes at the level of mediastinum in 30 cases and at the hilar level in three cases. The exact location of the target lesion by EBUS was obtained in 24 (73\%) patients. The main reasons for EBUS failure were, in one case only, a technical problem with the probe, and difficulty in obtaining good visualisation of lymph nodes, in particular around the trachea, mainly because of initial lack of EBUS experience and limitations due to local anaesthesia. Indeed, full inflation of the balloon in the large trachea is often followed by balloon detachment and, in this particular case, examination was continued with the probe directly applied on the wall. Under the latter conditions, the small size of the probe (relative to inflated balloon) often allows better demarcation of the sampling location, but adequate contact (and imaging) is only available in about three-quarters of the cases (fig. 1). EBUS localisation of lymph nodes required an additional time of $\sim 5 \mathrm{~min}$.

A total number of 139 samples (mean number per patient: $4.2 \pm 1.5$ ) were obtained in 39 different lymph node stations. The lymph node stations that were most frequently explored with TBNA were $4 \mathrm{R}(33.3 \%, 13$ patients) and $7(33.3 \%, 13$ patients), followed by $4 \mathrm{~L}(12.8 \%$, five patients), $11 \mathrm{R}(10.3 \%$, four patients), $11 \mathrm{~L}(5.1 \%$, two patients) and $10 \mathrm{~L}(2.6 \%$, one patient). In one case $(2.6 \%)$, it was unclear whether the target lesion was the primitive lesion located in close vicinity to the bronchial tree or contiguous agglomerated lymph nodes. Cytological sampling was obtained in 32 of the 33 patients $(97 \%)$, and tissue sampling in 23 patients $(70 \%)$. In two patients with non-neoplastic lymph node disorders, including one case of sarcoidosis, cytological sampling was not contributive, whereas tissue sampling allowed a diagnosis to be reached. In the case with anthracosis, follow-up until 8 months was also uneventful in supporting benign disease. Surprisingly, in one patient with small cell carcinoma, only tissue sampling was diagnostic.

Table 2 shows the final diagnosis, and the overall diagnostic yield of TBNA, as well as the diagnostic yield after previous localisation using EBUS. TBNA allowed diagnosis to be reached in $27(82 \%)$ of the patients, of whom $78 \%$ were diagnosed after previous EBUS localisation. Previous localisation with EBUS was associated with a TBNA diagnostic yield of $88 \%$ (21 out of 24) and failure of EBUS localisation with a diagnostic yield of $67 \%$ (six out of nine). A definite diagnosis was more often possible in case of neoplastic (25 out of 27, $92 \%$ ) than non-neoplastic involvement (two out of six, 33\%). In the six cases (18\%) without diagnosis after TBNA, lymph cells were observed in smears by the cytologist in only three cases. Further surgical sampling (mediastinoscopy) was performed in three of these six patients (two from group I and one from group III), and showed lymph node tissue without malignant involvement. In two additional cases, the multidisciplinary medical team considered that the lymph nodes were likely to be invaded (in one case, a synchronous advanced head and neck cancer was also discovered) and in the last case, clinical follow-up was chosen by the referring team and was uneventful at 20 months, supporting non-neoplastic disease. 


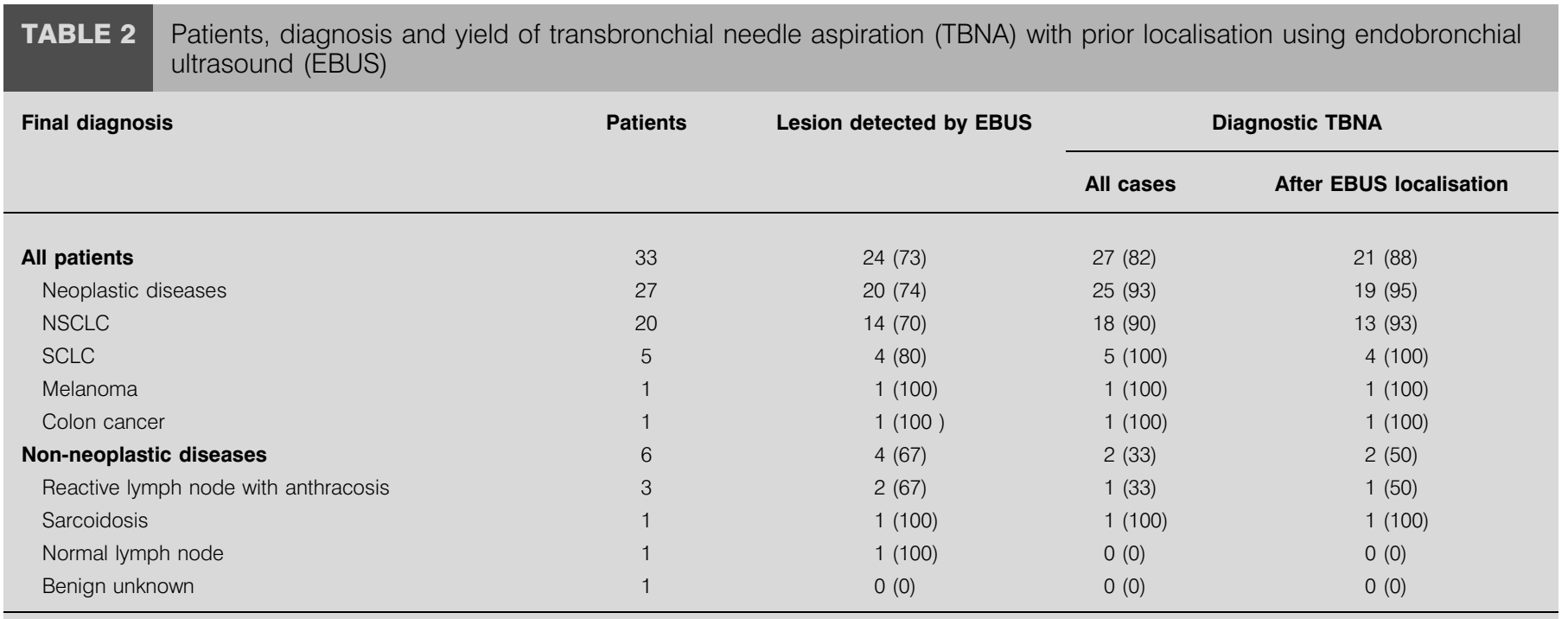

Data are presented as $n$ or $n(\%)$, unless otherwise indicated. NSCLC: nonsmall cell lung cancer; SCLC: small cell lung cancer.

Surgical sampling (mediastinoscopy or other surgical sampling of mediastinal lymph nodes) was avoided by the positive results of TBNA in 25 cases (76\% of the patients). In one case from group II (diagnosis and/or staging), an alternative procedure would have been possible in case of negative TBNA, namely a puncture of suspect liver metastasis.

\section{DISCUSSION}

The present study shows that TBNA combined with previous EBUS examination is a very safe and effective method with which to assess patients with FDG-PET positive lymph nodes. This technique could be performed under local anaesthetic, on a real outpatient basis and without any complication, and was associated with a high diagnostic yield. It also allowed avoidance of surgical diagnostic/staging sampling in $76 \%$ of the patients. To that extent, this technique should be considered as a primary method of histological sampling in patients with FDG-PET positive mediastinal lymph nodes. In case no lymph node metastases are demonstrated, however, due to low negative predictive value [5], further surgical sampling should be performed.

\section{Limitations of the study}

The initial diagnostic work-up of the patients was performed in various centres using different positron emission tomography (PET) and CT scans installations and different protocols. Accurate information on PET and CT scan results (standardised uptake value, lymph node measurements) were most often missing, however, the prospective inclusion of all referred patients and their multicentric origins guarantee that the population was not selected. Similarly, clinical decision after TBNA was taken by the referring centres and, even if recommended in case of negative TBNA results, mediastinal surgical sampling was performed in only three of the six cases.

TBNA use for the evaluation of lymph node involvement has been popularised by the works of WANG and coworkers [1315], but it remains largely underutilised, despite its safety, mainly because of concerns about the practice learning curve
[8]. This factor could also explain the wide range of diagnostic yields reported in the literature [5, 8]. Until recently, the procedure was performed without guidance and, in order to compensate for this drawback and to increase diagnostic yield, repeated aspirates, up to a total number of seven, and/or rapid on-site sample evaluation have been proposed [16]. The introduction of EBUS has recently led to major progress, and HERTH et al. [9] have shown that TBNA performed after EBUS localisation is better than conventional TBNA in patients with enlarged lymph nodes on CT scan. With regard to EBUSguided TBNA, prior evaluation with FDG-PET scan could offer two potential advantages. First, it may decrease the need for useless EBUS-guided TBNA in the case of a negative FDG-PET scan at the level of the mediastinum. Secondly, by a better selection of the lymph node stations that need to be sampled, it may increase TBNA diagnostic yield.

In the present study, the overall diagnostic yield of TBNA was $82 \%(93 \%$ in the case of neoplastic diagnosis). The current authors believe that these results are mainly due to the introduction of FDG-PET, allowing selection of positive lymph node station for EBUS localisation followed by TBNA. Prior evaluation and selection for lymph node sampling with FDGPET has also recently been advocated for oesophageal endoscopic ultrasound with fine needle aspiration $[17,18]$. In order to further assess whether FDG-PET effectively contributes to the high diagnostic yield in the present study, a randomised study comparing TBNA combined with EBUS, with or without prior evaluation with FDG-PET, should be required. One problem with such a study, however, is related to the fact that a PET scan has also been shown to afford significant additional information in the search for metastasis of lung cancer, as well as being cost-effective [4, 19].

One may also wonder whether, after prior selection of lymph nodes with CT as well as FDG-PET scan, previous lymph node localisation with EBUS will still increase the diagnostic yield of conventional TBNA. In the present study, a previous localisation with EBUS was associated with a diagnostic yield of $88 \%$ 
(21 out of 24), and failure of EBUS localisation with a diagnostic yield of $67 \%$ (six out of nine). This question, however, appears to be of low importance for at least two reasons. First, there will still remain many indications for lymph node histological sampling with TBNA in which prior evaluation with FDG-PET is not indicated or performed, such that TBNA combined with EBUS will still be required. Secondly, a new echo-bronchoscope allowing real-time guided TBNA has recently been developed and will probably decrease the number of samples required for diagnosis while, at the same time, increasing the diagnostic yield of the technique [20, 21]. In the current authors' opinion, the real-time guided TBNA will probably replace the ultrasonic probe technique used in the present study for localisation prior TBNA. However, one limitation of the real-time guided TBNA is related to the fact that it only allows cytological sampling.

In the present study, TBNA after EBUS localisation replaced $75 \%$ of the surgical diagnostic/staging procedures. These results are in accordance with previous studies showing that oesophageal endoscopic ultrasound with fine needle aspiration in lung cancer patients with mediastinal and/or upper retroperitoneal FDG-PET hot spots in fact decreased, by an amount of $62 \%$, the surgical staging procedures and saved $40 \%$ of staging costs [17]. This initial approach may also confer an advantage in the context of multi-modal treatment. Provided N2 disease is confirmed by initial TBNA sampling, re-staging after an induction treatment, which is of paramount importance to assess the important prognostic factor that is downstaging [22-24], might be performed with mediastinoscopy without the limitations that this examination shows when it follows a previous one (remediastinoscopy) [25, 26].

In summary, transbronchial needle aspiration after endobronchial ultrasound localisation is a primary technique for (cyto)histological sampling of mediastinal(hilar) lymph nodes, whose yield can be optimised with a proper selection of the target lesion using positron emission tomography with 18Ffluoro-2-deoxy-D-glucose. In this particular clinical setting, this procedure may reduce the need for invasive surgical procedures, including mediastinoscopy, in the majority of the cases. Mediastinoscopy is, however, still indicated in case of negative transbronchial needle aspiration.

\section{REFERENCES}

1 Mountain CF. Revisions in the international system for the staging of lung cancer. Chest 1977; 111: 1710-1717.

2 Watanabe Y. Surgical outcomes of N2 and T3-4 non-small cell lung cancer. Lung Cancer 2003; 42: Suppl. 2, S23-S28.

3 Toloza EM, Harpole L, McCrory DC. Noninvasive staging of non-small cell lung cancer: a review of the current evidence. Chest 2003; 123: Suppl. 1, 137S-146S.

4 Vansteenkiste JF, Stroobants SG. The role of positron emission tomography with 18F-fluoro-2-deoxy-D-glucose in respiratory oncology. Eur Respir J 2001; 17: 802-820.

5 Silvestri GA, Tanoue LT, Margolis ML, Barker J, Detterbeck F. American College of Chest Physicians. The noninvasive staging of non-small cell lung cancer: the guidelines. Chest 2003; 123: Suppl. 1, 147S-156S.

6 Luke WP, Pearson FG, Todd TRJ. Prospective evaluation of mediastinoscopy. J Thor Cardiovasc Surg 1986; 91: 53-56.
7 Schieppati E. La puncion mediastinal traves del espolon traquel. Rev As Med Argent 1949; 663: 497-499.

8 Haponik EF, Shure D. Underutilization of transbronchial needle aspiration. Experience of current pulmonary fellows. Chest 1997; 112: 251-253.

9 Herth F, Becker HD, Ernst A. Conventional vs endobronchial ultrasound-guided transbronchial needle aspiration: a randomized trial. Chest 2004; 125: 322-325.

10 Vermylen P, Pierard P, Roufosse C, et al. Detection of bronchial preneoplastic lesions and early lung cancer with fluorescence bronchoscopy: a study about its ambulatory feasibility under local anesthesia. Lung Cancer 1999; 25: 161-168.

11 Harkin TJ, Wang K. Bronchoscopic needle aspiration of mediastinal and hilar lymph nodes. J Bronchology 1997; 4: 238-249.

12 Mountain CF, Dresler CM. Regional lymph node classification for lung cancer staging. Chest 1997; 111: 1718-1723.

13 Wang KP, Terry PB. Transbronchial needle aspiration in the diagnosis and staging of bronchogenic carcinoma. Am Rev Respir Dis 1983; 127: 344-347.

14 Wang KP. Flexible transbronchial needle aspiration for histology specimen. Chest 1985; 88: 860-863.

15 Wang KP. Staging of bronchogenic carcinoma by bronchoscopy. Chest 1995; 106: 588-593.

16 Chin R, McCain TW, Lucla MA, et al. Transbronchial needle aspiration in diagnosing and staging lung cancer. How many aspirates are needed? Am J Respir Crit Care Med 2002; 166: 377-381.

17 Kramer H, van Putten JWG, Post WJ, et al. Oesophageal endoscopic ultrasound with fine needle aspiration improves and simplifies the staging of lung cancer. Thorax 2004; 59: 596-601.

18 Annema JT, Hoekstra OS, Smit EF, Veselic M, Versteegh MIM, Rabe KF. Towards a minimally invasive staging strategy in NSCLC: analysis of PET positive mediastinal lesions by EUS-FNA. Lung Cancer 2004; 44: 53-60.

19 van Tinteren H, Hoekstra OS, Smit EF, et al. Effectiveness of positron emission tomography in the preoperative assessment of patients with suspected non-small-cell lung cancer: the PLUS multicentre randomised trial. Lancet 2002; 359: 1388-1393.

20 Krasnik M, Vilman P, Larsen SS, Jacobsen GK. Preliminary experience with a new method of endoscopic transbronchial real time ultrasound guided biopsy for diagnosis of mediastinal and hilar lesions. Thorax 2003; 58: 1083-1086.

21 Yasufuku K, Chiyo M, Sekine Y, et al. Real-time endobronchial ultrasound-guided transbronchial needle aspiration of mediastinal and hilar lymph nodes. Chest 2004; 126: 122-128.

22 Bueno R, Richards WG, Swanson SJ, et al. Nodal stage after induction therapy for stage IIIA lung cancer determines patient survival. Ann Thorac Surg 2000; 70: 1826-1831.

23 Voltolini L, Luzzi L, Ghiribelli C, et al. Results of induction chemotherapy followed by surgical resection in patients with stage IIIA (N2) non-small cell lung cancer: the importance of the nodal down-staging after chemotherapy. Eur J Cardiothorac Surg 2001; 20: 1106-1112. 
24 Sawabata N, Keller SM, Matsumura O, et al. The impact of residual multi-level $\mathrm{N} 2$ disease after induction therapy for non-small cell lung cancer. Lung Cancer 2003; 42: 69-78.

25 Mateu-Navarro M, Rami-Porta R, Bastus-Piulats R, et al. Remediastinoscopy after induction chemotherapy in non-small cell lung cancer. Ann Thorac Surg 2000; 70: 391-395.

26 Van Schil P, van der School J, Poniewierski J. Remediastinoscopy after neoadjuvant therapy for nonsmall cell lung cancer. Lung Cancer 2002; 37: 281-285. 